\title{
Integrated analysis of differentially expressed genes in breast cancer pathogenesis
}

\author{
DAOBAO CHEN and HONGJIAN YANG \\ Department of Breast Surgery, Zhejiang Cancer Hospital, Hangzhou, Zhejiang 310022, P.R. China
}

Received October 20, 2014; Accepted March 10, 2015

DOI: $10.3892 / \mathrm{ol} .2015 .3147$

\begin{abstract}
The present study aimed to detect the differences between breast cancer cells and normal breast cells, and investigate the potential pathogenetic mechanisms of breast cancer. The sample GSE9574 series was downloaded, and the microarray data was analyzed to identify differentially expressed genes (DEGs). Gene Ontology (GO) cluster analysis using the GO Enrichment Analysis Software Toolkit platform and Kyoto Encyclopedia of Genes and Genomes (KEGG) pathway analysis for DEGs was conducted using the Gene Set Analysis Toolkit V2. In addition, a protein-protein interaction (PPI) network was constructed, and target sites of potential transcription factors and potential microRNA (miRNA) molecules were screened. A total of 106 DEGs were identified in the current study. Based on these DEGs, a number of bio-pathways appear to be altered in breast cancer, including a number of signaling pathways and other disease-associated pathways, as indicated by KEGG pathway clustering analysis. ATF3, JUND, FOSB and $J U N B$ were detected in the PPI network. Finally, the most significant potential target sites of transcription factors and miRNAs in breast cancer, which are important in the regulation of gene expression, were identified. The results indicated that miR-93, miR-302A, miR-302B, miR-302C, miR-302D, miR-372, miR-373, miR-520E and miR-520A were closely associated with the occurrence and development of breast cancer. Therefore, changes in the expression of these miRNAs may alter cell metabolism and trigger the development of breast cancer and its complications.
\end{abstract}

\section{Introduction}

Breast cancer is a type of cancer that originates from breast tissue and most commonly from the inner lining of the milk

Correspondence to: Dr Daobao Chen, Department of Breast Surgery, Zhejiang Cancer Hospital, 38 Guangji Road, Hangzhou, Zhejiang 310022, P.R. China

E-mail: daobaochendbc@163.com

Key words: differentially expressed genes, transcription factors, breast cancer, function and pathway annotation, protein-protein interaction network, microRNA ducts or from the lobules that supply the ducts (1). Breast cancer affects $\sim 1.2$ million women worldwide and accounts for $\sim 50,000$ mortalities every year (2). Despite major advances in surgical and nonsurgical management of the disease, breast cancer metastasis remains a significant clinical challenge affecting numerous of patients (3). The prognosis and survival rates for breast cancer are highly variable, and depend on the cancer type, treatment strategy, stage of the disease and geographical location of the patient (4).

Microarray technology, which may be used to simultaneously interrogate 10,000-40,000 genes, has provided new insight into the molecular classification of different cancer types (5). Many genes involved in the regulation of cell cycle, invasion, metastasis and angiogenesis have been indicated to be prognostic biomarkers based on microarray analyses. Furthermore, numerous researchers have proposed that the phenotypic diversity of breast tumors may be accompanied by a corresponding diversity in gene expression patterns (6). Therefore, the systematic investigation of gene expression patterns in human breast tumors may aid in understanding the pathogenesis of this disease (7).

The present study aimed to investigate the differences between breast cancer cells and normal cells, and examine the possible underlying mechanisms of breast cancer. Biological microarray analysis was used to analyze the expression profile of breast cancer and normal cells, and identify the differentially expressed genes (DEGs). In addition, altered metabolic pathways in breast cancer were identified using a bioinformatics approach, while the target sites of potential transcription factors and miRNAs were screened. Furthermore, the current study aimed to improve the understanding of the occurrence and development of breast cancer and facilitate the discovery of potential novel biomarkers for its treatment.

\section{Materials and methods}

Gene expression microarray. In order to investigate the alterations in breast cancer cells compared with normal cells, DEGs were screened at the gene level and the possible mechanisms were examined. The gene expression series, GSE9574 (8), was downloaded from the Gene Expression Omnibus (GEO; http:// www.ncbi.nlm.nih.gov/geo/) of the National Center for Biotechnology Information (Bethesda, MD, USA), based on the GPL96 [HG-U133] platform data (Affymetrix Human Genome U133 Array), and included 14 breast cancer and 15 normal samples. 
Identification of DEGs. Microarray data were analyzed using R software v.2.13.0 (9) and further processed using Geoquery (10) and Limma (11) packages. Geoquery is used to rapidly obtain gene expression profiles from the GEO database (10), while the Limma package is the most popular method for the analysis of DEGs $(11,12)$. The preprocessed expression data were obtained using Geoquery, and subjected to a $\log _{2}$ transformation. The breast cancer and normal samples were compared using Limma in order to identify the DEGs between the two tissue types. Gene P-values were determined by $\mathrm{R}$ software using the Student's $t$-test and $\mathrm{P}<0.001$ in a Bayesian model was considered to indicate a DEG (12).

Gene Ontology (GO) analysis of DEGs. In order to assess the changes in DEGs occurring at the cellular level and the functional clustering of DEGs, the GO Enrichment Analysis Software Toolkit (GOEAST) (13) in the GO database (14) was used. Hypergeometric algorithms were used for statistical analysis, and terms associated with biological process and molecular function were enriched.

Bio-pathway analysis of DEGs. In order to detect the changes in DEGs at the molecular level, all the metabolic and nonmetabolic pathways were obtained from the Kyoto Encyclopedia of Genes and Genomes (KEGG) database. Pathway enrichment of DEGs was assessed using the Gene Set Analysis Toolkit v2 $(15,16)$, and the number of genes was counted for each term. A gene number of $\geq 2$ and $\mathrm{P}<0.05$ were used as the cut-off values.

Protein-protein interaction (PPI) network construction. PPI data were integrated and verified using data obtained from the following databases (all accessed on November 11, 2012): Human Protein Reference Database (http://www.hprd. org/); Biological General Repository for Interaction Datasets (http://thebiogrid.org/); Biomolecular Object Network Databank (http://bond.unleashedinformatics.com/); Database of Interacting Proteins (http://dip.doe-mbi.ucla.edu/); IntAct (http://www.ebi.ac.uk/intact/); Molecular INTeraction database (http://mint.bio.uniroma2.it/mint/welcome.do); and Reactome (http://www.reactome.org/). Subsequently, a PPI network was constructed based on the identified DEGs. A hypergeometric algorithm was used, and $\mathrm{P}<0.05$ was considered to indicate statistically significant differences.

Screening the target sites of potential transcription factors and miRNAs. Based on the gene annotation data from the Molecular Signatures Database (http://www.broadinstitute. org/gsea/msigdb/index.jsp; accessed November 11, 2012), the abundance of the gene sets were analyzed. In addition, hypergeometric algorithms and false discovery rate (FDR) correction were performed using the Benjamini \& Hochberg method (17). FDR $<0.05$ was selected as the statistical significance threshold to indicate target sites of potential transcription factors and miRNAs.

\section{Results}

Identification of DEGs in breast cancer. Using $\mathrm{P}<0.001$ as the statistical significance threshold, a total of 123 probes were identified, which presented altered expression levels in breast

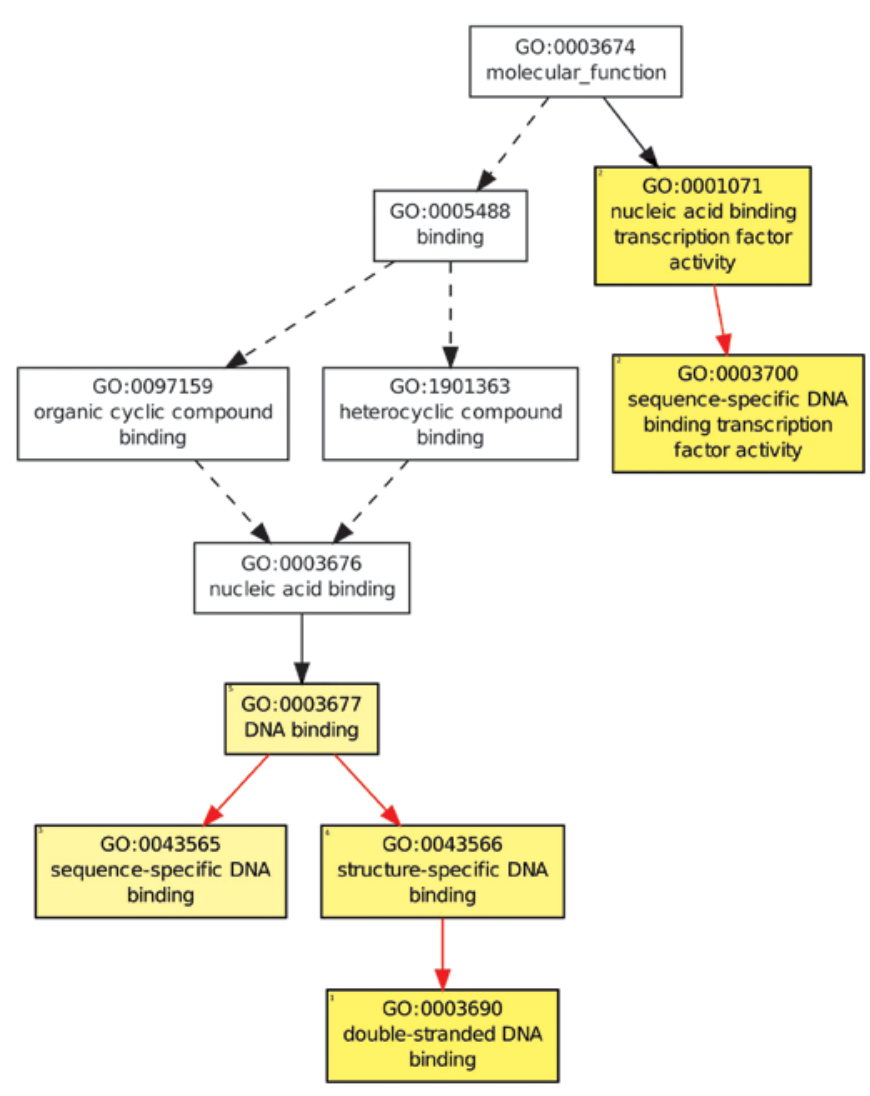

Figure 1. Molecular function clustering of differentially expressed genes Yellow boxes are significantly enriched Gene Ontology terms (false discovery rate $<0.051)$. Brighter color indicates stronger statistical significance. GO, Gene Ontology.

cancer when compared with normal tissues, and involved 106 DEGs (Table I).

GO cluster of DEGs. The molecular functions enriched in the identified DEGs included nucleic acid binding transcription factor activity, sequence-specific DNA binding transcription factor activity and double-stranded DNA binding (Fig. 1). In addition, the biological processes enriched are shown in Fig. 2, and include positive regulation of biological process, positive regulation of cellular process, cellular response to organic substance and positive regulation of transcription from RNA polymerase II promoter. The GO clustering results provide a preliminary description of the potential functions of the DEGs and their effects on cells.

Bio-pathways altered in breast cancer. To further investigate changes of the biological pathways within cancer cells in detail, a KEGG pathway enrichment analysis of the identified DEGs was performed. KEGG clustering results indicated that a number of bio-pathways were altered in breast cancer cells, primarily signaling and disease-associated pathways (Table II). The alteration of the RNA transport pathway was consistent with the GO clustering results, suggesting that gene expression in breast cancer cells differs from that in normal cells. In addition, signaling pathways in the cell surface were altered, including the nucleotide-binding oligomerization domain (NOD)-like receptor signaling pathway, epithelial cell signaling in Helicobacter pylori 


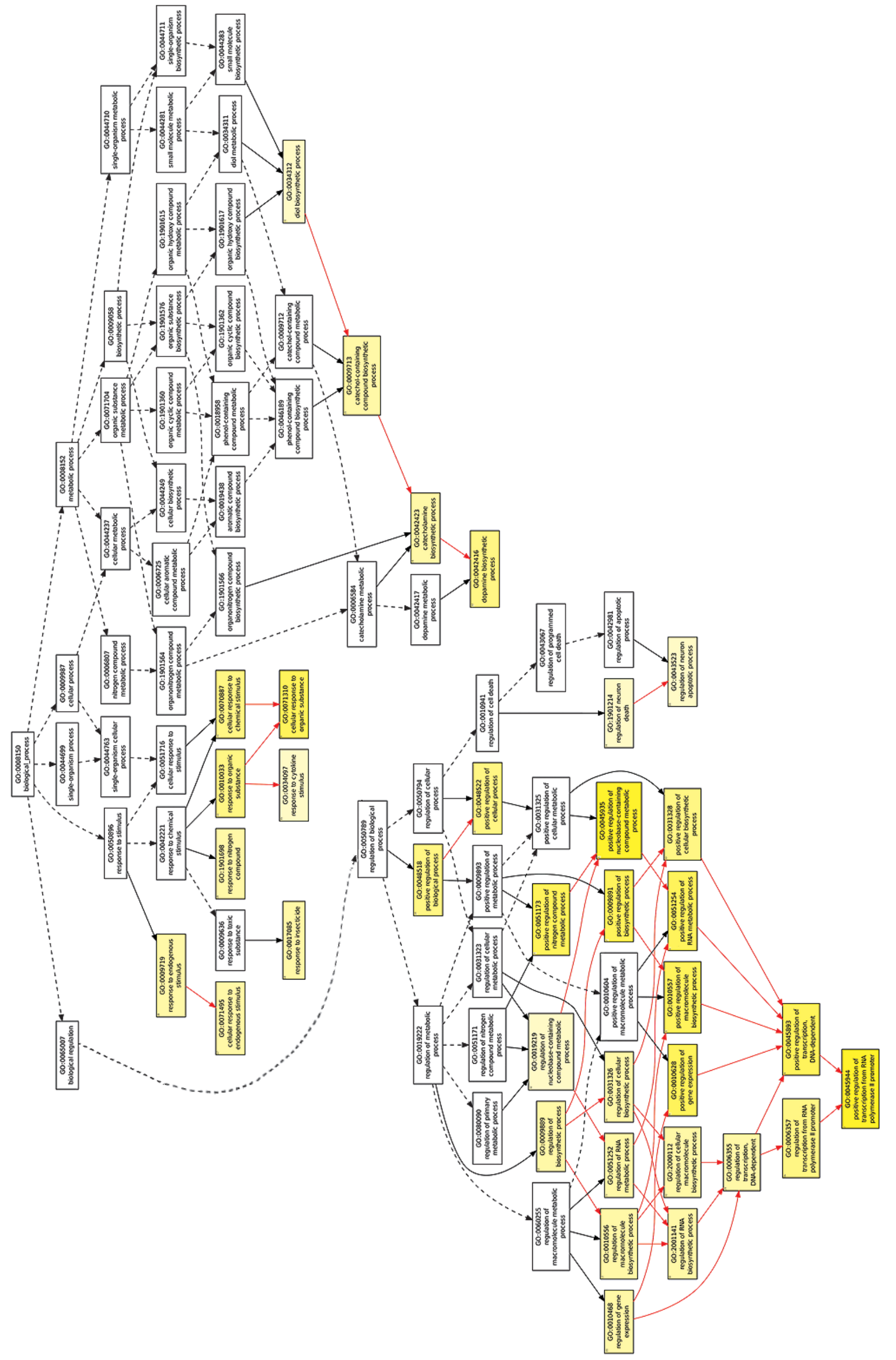


Table I. Differentially expressed genes in breast cancer tissue as compared with normal tissue.

\begin{tabular}{|c|c|c|c|}
\hline Gene symbol & P-value & Gene symbol & P-value \\
\hline PTP4A1 & $7.48 \times 10^{-9}$ & SAFB2 & $2.98 \times 10^{-4}$ \\
\hline IER2 & $1.10 \times 10^{-8}$ & SPATS2L & $3.01 \times 10^{-4}$ \\
\hline FOSB & $1.23 \times 10^{-8}$ & MST1P9 & $3.12 \times 10^{-4}$ \\
\hline NR4A3 & $4.97 \times 10^{-8}$ & CCL2 & $3.16 \times 10^{-4}$ \\
\hline ATF3 & $1.36 \times 10^{-7}$ & AKR7A2 & $3.34 \times 10^{-4}$ \\
\hline BTG2 & $1.61 \times 10^{-7}$ & LSR & $3.35 \times 10^{-4}$ \\
\hline NOL12 & $2.01 \times 10^{-7}$ & DNAJB4 & $3.38 \times 10^{-4}$ \\
\hline FOS & $2.21 \times 10^{-7}$ & KLF11 & $3.42 \times 10^{-4}$ \\
\hline TACSTD2 & $5.92 \times 10^{-7}$ & TP53TG1 & $3.60 \times 10^{-4}$ \\
\hline H3F3B & $9.46 \times 10^{-7}$ & GNAS & $3.79 \times 10^{-4}$ \\
\hline JUN & $1.00 \times 10^{-6}$ & CD69 & $3.90 \times 10^{-4}$ \\
\hline EIF1 & $1.17 \times 10^{-6}$ & NR4A1 & $3.92 \times 10^{-4}$ \\
\hline NR4A2 & $1.72 \times 10^{-6}$ & TIMM23 / TIMM23B & $4.22 \times 10^{-4}$ \\
\hline DUSP1 & $5.29 \times 10^{-6}$ & JUNB & $4.30 \times 10^{-4}$ \\
\hline EIF5 & $7.45 \times 10^{-6}$ & FGF20 & $4.49 \times 10^{-4}$ \\
\hline TGFB2 & $1.13 \times 10^{-5}$ & $\mathrm{HIC} 2$ & $4.51 \times 10^{-4}$ \\
\hline APOH & $1.31 \times 10^{-5}$ & GP6 & $4.53 \times 10^{-4}$ \\
\hline ZFP36 & $2.30 \times 10^{-5}$ & SIK1 & $4.77 \times 10^{-4}$ \\
\hline GPR183 & $2.34 \times 10^{-5}$ & PGS1 & $4.77 \times 10^{-4}$ \\
\hline JUND & $2.51 \times 10^{-5}$ & SIDT2 & $4.89 \times 10^{-4}$ \\
\hline EXOC7 & $2.71 \times 10^{-5}$ & IL33 & $5.04 \times 10^{-4}$ \\
\hline MCL1 & $2.73 \times 10^{-5}$ & FLRT2 & $5.05 \times 10^{-4}$ \\
\hline REXO4 & $3.17 \times 10^{-5}$ & TRAIP & $5.10 \times 10^{-4}$ \\
\hline CLDN1 & $3.69 \times 10^{-5}$ & PLXND1 & $5.21 \times 10^{-4}$ \\
\hline HEY2 & $4.63 \times 10^{-5}$ & YWHAZ & $5.55 \times 10^{-4}$ \\
\hline KLF6 & $5.98 \times 10^{-5}$ & LINC00094 & $5.60 \times 10^{-4}$ \\
\hline CYLD & $6.32 \times 10^{-5}$ & ZNF451 & $5.90 \times 10^{-4}$ \\
\hline SH3BP2 & $6.76 \times 10^{-5}$ & ZNF232 & $6.16 \times 10^{-4}$ \\
\hline SERPINE1 & $7.88 \times 10^{-5}$ & DUSP2 & $6.24 \times 10^{-4}$ \\
\hline HIST2H2BE & $8.21 \times 10^{-5}$ & EBLN2 & $6.31 \times 10^{-4}$ \\
\hline PRR5-ARHGAP8 / ARHGAP8 & $8.36 \times 10^{-5}$ & XPNPEP1 & $6.40 \times 10^{-4}$ \\
\hline EGR 1 & $9.68 \times 10^{-5}$ & FAM46C & $6.61 \times 10^{-4}$ \\
\hline KLF4 & $1.09 \times 10^{-4}$ & PDE4B & $6.77 \times 10^{-4}$ \\
\hline IL5RA & $1.24 \times 10^{-4}$ & RBM14 & $6.79 \times 10^{-4}$ \\
\hline PMAIP1 & $1.33 \times 10^{-4}$ & RGS1 & $6.83 \times 10^{-4}$ \\
\hline FANCG & $1.35 \times 10^{-4}$ & SLC33A1 & $7.28 \times 10^{-4}$ \\
\hline NOS1AP & $1.65 \times 10^{-4}$ & SLC35E2 & $7.34 \times 10^{-4}$ \\
\hline C1orf50 & $1.69 \times 10^{-4}$ & CNDP2 & $7.43 \times 10^{-4}$ \\
\hline MUM1 & $1.69 \times 10^{-4}$ & GTF2H1 & $7.64 \times 10^{-4}$ \\
\hline STK17B & $1.72 \times 10^{-4}$ & CARKD & $7.77 \times 10^{-4}$ \\
\hline ILF3 & $1.91 \times 10^{-4}$ & CCNL1 & $7.78 \times 10^{-4}$ \\
\hline CLNS1A & $2.01 \times 10^{-4}$ & CDC42 & $7.84 \times 10^{-4}$ \\
\hline BHLHE40 & $2.21 \times 10^{-4}$ & TAF1D & $8.08 \times 10^{-4}$ \\
\hline ATP2B2 & $2.30 \times 10^{-4}$ & CLGN & $8.22 \times 10^{-4}$ \\
\hline TSC22D2 & $2.31 \times 10^{-4}$ & SGSM2 & $8.23 \times 10^{-4}$ \\
\hline LAGE3 & $2.35 \times 10^{-4}$ & KIF1A & $8.48 \times 10^{-4}$ \\
\hline ZNHIT1 & $2.25 \times 10^{-4}$ & NRAS & $8.87 \times 10^{-4}$ \\
\hline CXCL2 & $2.60 \times 10^{-4}$ & KLF10 & $8.96 \times 10^{-4}$ \\
\hline UNC119B & $2.62 \times 10^{-4}$ & ORC5 & $9.17 \times 10^{-4}$ \\
\hline C9orf3 & $2.78 \times 10^{-4}$ & CCDC170 & $9.41 \times 10^{-4}$ \\
\hline GBAP1 & $2.85 \times 10^{-4}$ & C14orf105 & $9.54 \times 10^{-4}$ \\
\hline TTC38 & $2.86 \times 10^{-4}$ & MORC2 & $9.74 \times 10^{-4}$ \\
\hline IL8 & $2.86 \times 10^{-4}$ & EIF4A1 & $9.99 \times 10^{-4}$ \\
\hline
\end{tabular}

Table I. Continued. 
Table II. Bio-pathways altered in breast cancer.

\begin{tabular}{ll}
\hline KEGG pathway & P-value \\
\hline MAPK signaling pathway & 0.0006 \\
Chagas disease (American trypanosomiasis) & 0.0006 \\
Osteoclast differentiation & 0.0009 \\
Rheumatoid arthritis & 0.0048 \\
Renal cell carcinoma & 0.0202 \\
NOD-like receptor signaling pathway & 0.0432 \\
GnRH signaling pathway & 0.0432 \\
Pathogenic Escherichia coli infection & 0.0432 \\
Malaria & 0.0432 \\
T cell receptor signaling pathway & 0.0432 \\
Chemokine signaling pathway & 0.0441 \\
Colorectal cancer & 0.0445 \\
Epithelial cell signaling in & 0.0445 \\
Helicobacter pylori infection & \\
Neurotrophin signaling pathway & 0.0445 \\
Pathways in cancer & 0.0445 \\
Leishmaniasis & 0.0475 \\
RNA transport & 0.0475 \\
B-cell receptor signaling pathway & 0.0475 \\
\hline
\end{tabular}

KEGG, Kyoto Encyclopedia of Genes and Genomes; NOD, nucleotide-binding oligomerization domain.

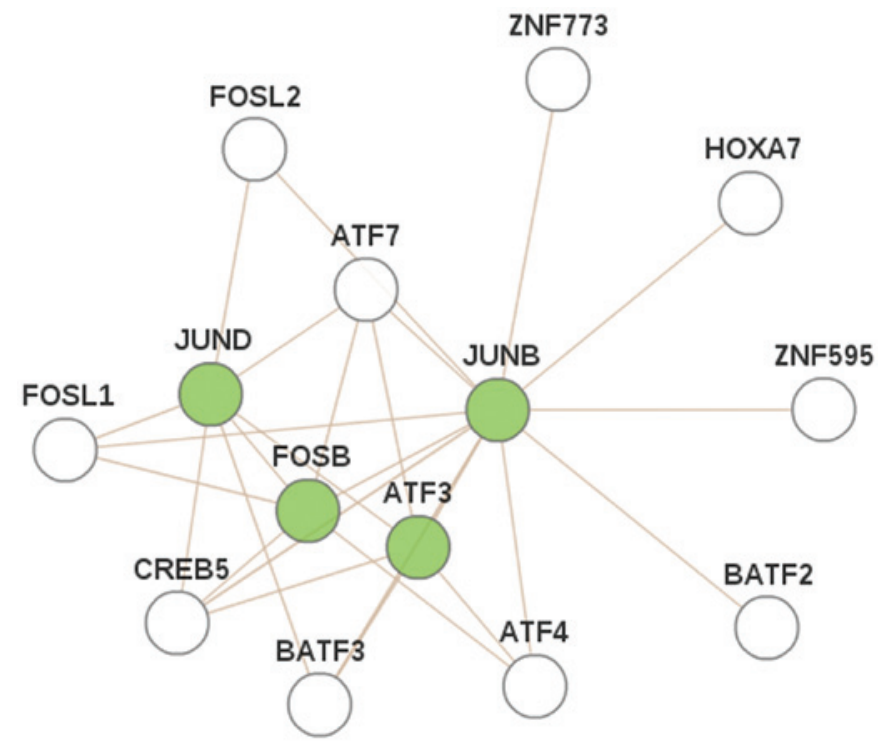

Figure 3. Protein-protein interaction network of differentially expressed genes (green circles) in breast cancer.

infection, chemokine signaling pathway, $\mathrm{T}$ cell receptor signaling pathway and B cell receptor signaling pathway. The latter three are involved in immune reactions, while the NOD-like receptor signaling pathway is closely associated with cell differentiation and development. Alterations in other metabolic pathways were also indicated, including the mismatch repair pathway, proteasome, metabolic pathways, glutathione metabolism and glycolysis/gluconeogenesis. Notably, KEGG clustering results indicated that a number of other disease pathways may also be altered, including Chagas disease (American trypanosomiasis), osteoclast differentiation, rheumatoid arthritis, renal cell carcinoma, pathogenic Escherichia coli infection, malaria, colorectal cancer, pathways in cancer and leishmaniasis.

Construction and analysis of PPI network. Four DEGs (JUND, $J U N B, F O S B$ and ATF3) were found to exhibit close associations with other genes, via the proteins identified to construct the PPI network (Fig. 3). These results show that the four DEGs interact indirectly, indicating that they may exhibit a joint role in the pathogenesis of breast cancer.

Screening of target sites of potential transcription factors. The expression of transcription factors affects the regulation downstream gene expression. Exploring the target sites of possible transcription factors is of great importance. The target sites of potential transcription factors were explored, and the ten most significant sites are listed in Table III. As shown in Table III, FOSB, ATF3 and JUND shared the same binding sites [Homo sapiens (hsa)_V\$ATF_01 and hsa_V\$ATF3_Q6]. Therefore, the regulation of these binding sites may present a potential treatment for breast cancer by controlling the expression of FOSB, ATF3 and JUND.

Screening of potential miRNAs. miRNAs regulate gene expression by controlling the stability of RNA. Therefore, identifying the regulatory miRNAs of specific DEGs may increase understanding with regard to the involvement of miRNAs in breast cancer. Potential regulatory miRNAs were analyzed based on the sequences of the DEGs, and the target sites with P-values of $<0.05$ are listed in Table IV. The regulatory miRNAs of two target sites, hsa_AGCACTT and hsa_ACTTTAT, were collected, which included miR-93, miR-302A, miR-302B, miR-302C, miR-373 and miR-520. These miRNAs may present potential therapeutic targets for breast cancer treatment.

\section{Discussion}

Breast cancer, similar to other cancer types, occurs due to an interaction between the environment and a defective gene (3). The incidence of breast cancer is increasing rapidly in the majority of Asian countries (18). Worldwide, breast cancer accounts for $22.9 \%$ of all cancer cases (excluding nonmelanoma skin cancers) in females. In 2008, breast cancer was the cause of 458,503 mortalities worldwide (13.7\% of cancer-associated mortalities in females) (2). Therefore, the study of breast cancer has important significance for human health. In the present study, a total of 106 DEGs between breast cancer and normal cells were identified. With regard to molecular function, the identified DEGs were predominantly involved in DNA-binding and regulation of downstream gene expression, as well as in metabolic and synthetic pathways. In terms of bio-pathways, signaling-associated pathways and other disease-associated pathways were found to be altered. ATF3, JUND, FOSB and $J U N B$ were the only four genes composing the PPI network of DEGs. Finally, the most significant target sites of potential transcription factors and miRNAs in breast cancer were identified. 
Table III. Target sites of potential transcription factors.

\begin{tabular}{lccc}
\hline Target & P-value & Gene count & \multicolumn{1}{c}{ Gene symbols } \\
\hline hsa_V\$CREB_Q2 & $3.51 \times 10^{-7}$ & 20 & GNAS, EGR1, CDC42, CYLD, FOSB, ATF3, FOS \\
hsa_V\$CREB_02 & $1.18 \times 10^{-6}$ & 16 & C9orf3, FOSB, NR4A2, DUSP1, JUND \\
hsa_V\$CREB_Q4 & $9.21 \times 10^{-6}$ & 15 & GNAS, CDC42, EIF1, NR4A2, ATF3, FOS \\
hsa_V\$ATF_01 & $9.21 \times 10^{-6}$ & 15 & EIF1, FOSB, NR4A2, ATF3, SIK1, FOS, JUND \\
hsa_V\$E4F1_Q6 & $1.10 \times 10^{-5}$ & 16 & GNAS, NR4A2, EIF1, FOSB, EIF4A1, HIC2 \\
hsa_V\$TAXCREB_01 & $1.10 \times 10^{-5}$ & 10 & CYLD, CDC42, FOSB, FOS, JUND, DUSP1 \\
hsa_GTGACGY_V\$E4F1_Q6 & $2.86 \times 10^{-5}$ & 22 & GNAS, PTP4A1, NR4A3, EGR1, JUNB, EIF1, JUND \\
hsa_V\$CREB_Q2_01 & $4.56 \times 10^{-5}$ & 16 & JUN, CYLD, EGR1, FOSB, NR4A1, DUSP1 \\
hsa_V\$SRF_C & $4.56 \times 10^{-5}$ & 13 & DUSP2, IER2, EGR1, JUNB, FOSB, KLF6, FOS \\
hsa_V\$ATF3_Q6 & $1.00 \times 10^{-4}$ & 13 & JUN, CYLD, CDC42, FOSB, ATF3, JUND
\end{tabular}

hsa, Homo sapiens; V\$, separator between transcription factor and its binding sequence.

Table IV. Potential miRNAs of differentially expressed genes with $\mathrm{P}<0.05$.

\begin{tabular}{lll}
\hline Target sequence & \multicolumn{1}{c}{ Potential miRNAs } \\
\hline hsa_AGCACTT & $\begin{array}{l}\text { miR-93, miR-302A, miR-302B, miR-302C, miR-302D, miR-372, miR-373, } \\
\text { miR-520E, miR-520A, miR-526B, miR-520B, miR-520C, miR-520D }\end{array}$ \\
hsa_ACTTTAT & miR-142-5P & 0.0182
\end{tabular}

miR or miRNA, microRNA; hsa, Homo sapiens.

Functionally, the differential expression of genes results in a variety of abnormal physiological processes, including alterations in gene expression regulation, RNA transcription and protein translation processes $(19,20)$. In the present study, changes in the RNA transport pathway were consistent with the GO clustering results, indicating that gene expression in breast cancer cells differs from that in normal cells. In addition, by KEGG clustering, a number of bio-pathways were revealed to be altered in breast cancer. Among the altered cell surface signaling pathways, alteration of the NOD-like receptor signaling pathway may be an important mechanism involved in the abnormal differentiation of breast cancer cells. By contrast, changes associated with the immune response, including the $\mathrm{T}$ cell receptor, the chemokine and the B-cell receptor signaling pathways, may be involved in the evasion of autoimmunity of breast cancer cells. In addition, the MAPK signaling pathway, which was also enriched, transports extracellular signals into the intracellular environment, and may affect downstream gene expression in breast cancer cells (21). Alterations in the aforementioned pathways may result in changes to a number of other metabolic signaling pathways, including the GnRH and the neurotrophin signaling pathways. In addition, differences in the mismatch repair pathway indicated that the self-repair capacity may be affected. Changes in proteasome, metabolic pathways, glutathione metabolism and glycolysis/gluconeogenesis further confirmed the results of the GO clustering analysis, which indicated that the metabolic capability of breast cancer cells was altered compared with that of normal cells.
ATF3 is a member of the mammalian activation transcription factor/cyclic adenosine monophosphate responsive element-binding protein family, which is induced by numerous signals in cancer tissues (22). The ATF3 product forms the activator protein 1 complex by interacting with the protein products of $J U N D, F O S B$ and $J U N B$, thus regulating the expression of downstream genes in response to cytokines, growth factors and cell stress (23). Certain studies have demonstrated that it may be involved in the resistance to p53-dependent cellular senescence and apoptosis (24). Therefore, the existence of the PPI network formed by ATF3, JUND, FOSB and JUNB may result in the inability of the p53 pathway to kill breast cancer cells.

Of the screened transcription factors, the majority are associated with cylindromatosis, cell division cycle 42, FOSB, nuclear receptor subfamily 4 and JUND. Among these genes, $J U N D$ has been proposed to protect cells from p53-dependent senescence and apoptosis (25), while FOSB has been implicated as a regulator of cell proliferation, differentiation and transformation (26) These genes are key factors in the pathogenesis of cancer, and the identification of associated transcription factors may help to elucidate the underlying mechanisms of breast cancer.

In the current study, miR-93 was identified as a potential regulatory miRNA of the DEGs in breast cancer. It has been reported that miR-93 is the substrate of caspase-3 during apoptosis (27). In the present study, miR-93 was found to be a potential tumor suppressor using bioinformatics. In addition, miR-302A has been strongly linked with the hypoxia 
pathway and is upregulated in response to hypoxia-inducible factors (28). Furthermore, this miRNA is also overexpressed in breast tumor cells and was found to be a potential regulatory miRNA of certain DEGs in breast cancer in the current study. miR-302B, a frequently amplified miRNA, is associated with intrahepatic metastasis of hepatocellular carcinoma (29). A previous study identified that miR-302C suppresses the expression of the prostate-specific antigen and prostate cancer cell proliferation (30). The results of the present study suggested that the role of miR-302C in breast cancer was consistent with its role in prostate cancer. Furthermore, a previous study has demonstrated that miR-373 is involved in the development of normal and cancer cells (31). In breast cancer tissues, miR-373 (31) is downregulated compared with normal breast tissues, which is consistent with the results of the current study. miR-520 is a short RNA molecule (32), with only a limited number of studies investigating its role in cancer; however, the present study identified that it may be involved in breast cancer.

In conclusion, a large number of DEGs in breast cancer share the same transcription factors and miRNAs. The target sites of these molecules may be important in the regulation of DEG expression. Therefore, regulating the expression of these genes through the aforementioned target sites may contribute towards the development of novel treatments for breast cancer.

\section{References}

1. Sariego J: Breast cancer in the young patient. Am Surg 76: 1397-1400, 2010.

2. Boyle P and Levin B (eds): World Health Organization: World Cancer Report 2008. IARC Press, Lyon, pp42-43, 2008.

3. Al-Hajj M, Wicha MS, Benito-Hernandez A, Morrison SJ and Clarke MF: Prospective identification of tumorigenic breast cancer cells. Proc Natl Acad Sci USA 100: 3983-3988, 2003.

4. Sant M, Allemani C, Capocaccia R, Hakulinen T, Aareleid T, Coebergh JW, Coleman MP, Grosclaude P, Martinez C, Bell J, et al; EUROCARE Working Group: Stage at diagnosis is a key explanation of differences in breast cancer survival across Europe. Int J Cancer 106: 416-422, 2003.

5. Sotiriou C, Neo SY, McShane LM, Korn EL, Long PM, Jazaeri A, Martiat P, Fox SB, Harris AL and Liu ET: Breast cancer classification and prognosis based on gene expression profiles from a population-based study. Proc Natl Acad Sci USA 100: 10393-10398, 2003.

6. Perou CM, Sørlie T, Eisen MB, et al: Molecular portraits of human breast tumours. Nature 406: 747-752, 2000.

7. Hu Z, Fan C, Oh DS, Marron JS, He X, Qaqish BF, Livasy C, Carey LA, Reynolds E, Dressler L, et al: The molecular portraits of breast tumors are conserved across microarray platforms. BMC Genomics 7: 96, 2006.

8. Tripathi A, King C, de la Morenas A, Perry VK, Burke B, Antoine GA, Hirsch EF, Kavanah M, Mendez J, Stone M, et al Gene expression abnormalities in histologically normal breast epithelium of breast cancer patients. Int J Cancer 122: $1557-1566,2008$

9. R Development Core Team: R: a language and environment for statistical computing. http://www.R-project.org. The R Foundation for Statistical Computing. Vienna, Austria, 2013.

10. Davis S and Meltzer PS: GEOquery: a bridge between the Gene Expression Omnibus (GEO) and BioConductor. Bioinformatics 23: 1846-1847, 2007.

11. Diboun I, Wernisch L, Orengo CA and Koltzenburg M: Microarray analysis after RNA amplification can detect pronounced differences in gene expression using limma. BMC Genomics 7: 252, 2006.

12. Smyth GK: Linear models and empirical bayes methods for assessing differential expression in microarray experiments Stat Appl Genet Mol Biol 3: e3, 2004.
13. Zheng Q and Wang XJ: GOEAST: a web-based software toolkit for Gene Ontology enrichment analysis. Nucleic Acids Res 36: W358-W363, 2008.

14. Ashburner M, Ball CA, Blake JA, Botstein D, Butler H, Cherry JM, Davis AP, Dolinski K, Dwight SS, Eppig JT, et al; The Gene Ontology Consortium: Gene ontology: Tool for the unification of biology. Nat Genet 25: 25-29, 2000.

15. Zhang B, Kirov S and Snoddy J: WebGestalt: an integrated system for exploring gene sets in various biological contexts. Nucleic Acids Res 33: W741-W748, 2005.

16. Duncan D, Prodduturi N and Zhang B: WebGestalt2: an updated and expanded version of the Web-based Gene Set Analysis Toolkit. BMC Bioinformatics 11: p10, 2010.

17. Benjamini Y and Hochberg Y: Controlling the false discovery rate: a practical and powerful approach to multiple testing. J R Statist Soc B 57: 289-300, 1995.

18. Tavassoli FA and Devilee P (eds): World Health Organization Classification of Tumours: Pathology and Genetics of Tumours of the Breast and Female Genital Organs. IARC Press, Lyon, France, pp116-119, 2003.

19. Thomas EA, Coppola G, Desplats PA, et al: The HDAC inhibitor $4 \mathrm{~b}$ ameliorates the disease phenotype and transcriptional abnormalities in Huntington's disease transgenic mice. Proc Natl Acad Sci USA 105: 15564-15569, 2008.

20. Greenbaum D, Colangelo C, Williams K and Gerstein M: Comparing protein abundance and mRNA expression levels on a genomic scale. Genome Biol 4: 117, 2003.

21. Menendez JA, Vellon L, Mehmi I, Teng PK, Griggs DW and Lupu R: A novel CYR61-triggered 'CYR61-alphavbeta3 integrin loop' regulates breast cancer cell survival and chemosensitivity through activation of ERK1/ERK2 MAPK signaling pathway. Oncogene 24: 761-779, 2005.

22. Gilchrist M, Thorsson V, Li B, Rust AG, Korb M, Roach JC, Kennedy K, Hai T, Bolouri H and Aderem A: Systems biology approaches identify ATF3 as a negative regulator of Toll-like receptor 4. Nature 441: 173-178, 2006.

23. Shaulian E and Karin M: AP-1 as a regulator of cell life and death. Nat Cell Biol 4: E131-E136, 2002.

24. Malkin D, Li FP, Strong LC, Fraumeni JF Jr, Nelson CE, Kim DH, Kassel J, Gryka MA, Bischoff FZ, Tainsky MA, et al: Germ line p53 mutations in a familial syndrome of breast cancer, sarcomas, and other neoplasms. Science 250: 1233-1238, 1990.

25. Sharma SC and Richards JS: Regulation of AP1 (Jun/Fos) factor expression and activation in ovarian granulosa cells. Relation of JunD and Fra2 to terminal differentiation. J Biol Chem 275: 33718-33728, 2000.

26. McClung CA and Nestler EJ: Regulation of gene expression and cocaine reward by CREB and DeltaFosB. Nat Neurosci 6: 1208-1215, 2003.

27. Yeung ML, Yasunaga J, Bennasser Y, Dusetti N, Harris D, Ahmad N, Matsuoka M and Jeang KT: Roles for microRNAs, miR-93 and miR-130b, and tumor protein 53-induced nuclear protein 1 tumor suppressor in cell growth dysregulation by human T-cell lymphotrophic virus 1 . Cancer Res 68 : 8976-8985, 2008

28. Card DAG, Hebbar PB, Li L, Trotter KW, Komatsu Y, Mishina Y and Archer TK: Oct4/Sox2-regulated miR-302 targets cyclin D1 in human embryonic stem cells. Mol Cell Biol 28: 6426-6438, 2008

29. Suh M-R, Lee Y, Kim JY, Kim SK, Moon SH, Lee JY, Cha KY, Chung HM, Yoon HS, Moon SY, et al: Human embryonic stem cells express a unique set of microRNAs. Dev Biol 270: 488-498, 2004

30. Greco SJ and Rameshwar P: MicroRNAs regulate synthesis of the neurotransmitter substance $P$ in human mesenchymal stem cell-derived neuronal cells. Proc Natl Acad Sci USA 104: 15484-15489, 2007.

31. Huang Q, Gumireddy K, Schrier M, le Sage C, Nagel R, Nair S, Egan DA, Li A, Huang G, Klein-Szanto AJ, et al: The microRNAs miR-373 and miR-520c promote tumour invasion and metastasis. Nat Cell Biol 10: 202-210, 2008.

32. Keklikoglou I, Koerner C, Schmidt C, Zhang JD, Heckmann D, Shavinskaya A, Allgayer H, Gückel B, Fehm T, Schneeweiss A, et al: MicroRNA-520/373 family functions as a tumor suppressor in estrogen receptor negative breast cancer by targeting NF- $\kappa \mathrm{B}$ and TGF- $\beta$ signaling pathways. Oncogene 31: 4150-4163, 2012. 